\title{
AN IMAGE SMEARING TECHNIQUE TO IMPROVE PIGEON HOLE IMAGING (PHI)
}

\author{
Kamila Afroj ${ }^{1}$ and K Siddique-e Rabbani \\ Department of Biomedical Physics \& Technology \\ University of Dhaka, Dhaka, Bangladesh \\ email: q.kamila@gmail.com,rabbani@univdhaka.edu
}

\begin{abstract}
This new imaging modality arises as an extension of the 6-electrode Focused Impedance Method (FIM) developed in our laboratory earlier. Current is driven through two concentric pairs of orthogonally placed electrodes while several potential electrodes are located along a diagonal centrally. Through a combination of current drives and potential measurements across adjacent diagonal electrodes, it is possible to obtain admittance sums along the rows and columns of a square matrix formed by these diagonally placed potential electrodes. Through simple backprojection along the rows and columns it is possible to reconstruct a crude image in a square matrix form. Due to its resemblance, the name Pigeon Hole Imaging (PHI) has been coined. The concept has been experimentally tested to identify the location of a single target object in a background of uniform conductivity in $2 \mathrm{D}$ and 3D phantoms, in 2x2 and $6 \times 6$ matrix formations respectively. Taking the highest pixel to represent the object position the images were correct in all the pixels of the $2 \times 2$ image but in only 16 of the 36 pixels adjacent to the diagonal electrodes in the $6 \times 6$ image. However, a systematic pattern of image shift was observed in most of the other pixels in the latter, based on which a set of rules were made to correct the image in another 14 pixels. Thus excepting the furthest 6 pixels, correct image could be obtained in all 30 pixels. Thus PHI will be useful in locating exact positions of single target objects simply.
\end{abstract}

Keywords: Electrical admittance, Pigeon Hole imaging, Electrical impedance, Focused impedance

\section{INTRODUCTION:}

Tetrapolar Impedance Measurement (TPIM) is the basis of most electrical impedance measurements for biological applications. In a volume conductor the zone of sensitivity of this measurement is rather wide and the measured value includes the effects of other organs in the vicinity. A significant development in impedance measurements was the introduction of Electrical Impedance Tomography (EIT) which provides images of tissue impedance distribution [1],[2]. Although the resolution offered by EIT is poor (about 10\% for a 16-electrode system) compared with other imaging modalities, for studies of large organs like heart, lungs and stomach, even such degree of resolution is often not required. Besides, EIT also has a significant contribution from objects in the $3^{\text {rd }}$ dimension with respect to the electrode plane in a complex way which renders the pixel image values inaccurate [3],[4]. It is typical to mark out a large region of interest in EIT image to represent a large organ, sum all the pixels within this region at different times to study the temporal behaviour of the organ. Therefore, it appears that technologies bridging the intermediate region may have useful applications. With this in mind, a new method named as Focused Impedance Method (FIM) was developed earlier in the Biomedical Physics Laboratory of University of Dhaka [5],[6], which essentially is a combination of two TPIM systems placed orthogonally, centred around the zone of interest. The sum of the impedances from these two orthogonal measurements results in a greater contribution from the central zone of interest, thus contributing to a focusing effect. Again, by placing two potential measuring electrodes diagonally in the central region, the requirement of the number of electrodes was reduced from 8 to 6 . The 6-electrode FIM was successful in experimental measurements on phantoms and on the human body for gastric emptying [6] and lung ventilation studies [7].

\footnotetext{
${ }^{1}$ Institute of Nuclear Medicine and Ultrasound, Bangladesh Atomic Energy Commission, Dhaka
} 
Pigeon Hole Imaging (PHI) came almost naturally as an extension of the above 6-electrode FIM. By increasing the number of diagonally placed potential measuring electrodes in the above mentioned FIM and employing a simple method of backprojection, a new imaging modality could be developed. 3 diagonal electrodes gave a $2 \times 2$ matrix image, 4 electrodes gave a $3 \times 3$ matrix image and so on. This method was experimentally studied in $2 \mathrm{D}$ and $3 \mathrm{D}$ phantoms for $2 \times 2$ and $6 \times 6$ matrices to locate the position of a single object, and results indicated successful position indication for all the pixels in the $2 \times 2$ matrix, but for only 16 pixels for the $6 \times 6$ matrix [8]. The study was extended further to increase the understanding, and to see if techniques can be developed to correct the image positions for the other pixels, which is the work presented here. As indicated above, for the larger 6x6 matrix, the 20 image pixels away from the electrode diagonal came out slightly displaced. Observing the pixel values of the images closely at different contrast values, a systematic pattern of shift could be identified, based on which a set of correction rules were obtained to correct the images in most other pixels. Thus 14 more pixels excepting the furthest 6 pixels could be imaged correctly, making a total of 20 pixels for a $6 \times 6$ matrix. This increases the usefulness of PHI. Thus PHI may be useful in determining the exact location of a single organ at shallow depths under the skin whose position is approximately known. Once localized, further studies and monitoring may be performed using FIM or other techniques. Besides, in cases where an organ moves slightly with time, PHI may be used to monitor the movement, and to obtain a better impedance value through a suitable combination of values in several pixels.

\section{MATERIALS \& METHODS}

\subsection{Concept of PHI}

For conceptual understanding, the method of PHI is explained for an example having four diagonal potential electrodes contributing to a $3 \times 3$ matrix. This may easily be extended to other matrix sizes. In figure 1, current is passed vertically and horizontally through the opposite pairs of outer electrodes, shown by dark circles. The four diagonal potential measuring electrodes are shown by white circles. The

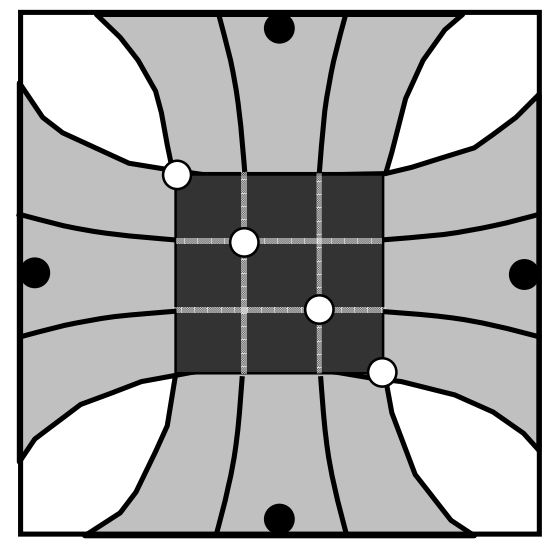

Fig. 1: Basic electrode arrangement for PHI. Dark dots representing electrodes indicate two orthogonal current driving pairs while white dots represent potential measuring electrodes.

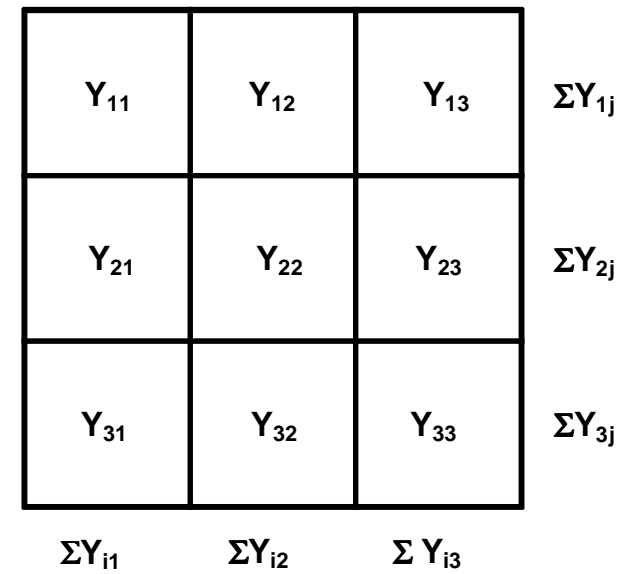

Fig. 2: Admittance model for the central zone. The sums of the rows and columns are the measured parameters.

nearly horizontal curved lines passing through the four diagonal electrodes represent equipotential lines when current is passed though the vertical pair of current electrodes. Similarly the nearly vertical curved lines passing through the four diagonal electrodes represent equipotential lines when current is passed though the horizontal pair of current electrodes. Let the dark square region in the centre shown in figure 1 
be represented as a matrix of 9 admittances as shown in figure 2. For simplicity, the segments of the equipotential lines forming the $3 \times 3$ matrix in the centre are assumed straight lines. It is assumed that these admittances are isotropic, and that the region outside the dark square in figure 1 has negligible contribution to the measurements performed.

When current is passed through the vertical pair of current electrodes and potential is measured between the top two diagonal electrodes the admittance measured would be given by

$$
\Sigma \mathrm{Y}_{1 \mathrm{j}}=\mathrm{Y}_{11}+\mathrm{Y}_{12}+\mathrm{Y}_{13} \text {, }
$$

as a parallel combination of the individual admittances along the top row as shown in figure 2, neglecting the contributions of the outer regions as assumed above. With the same current electrodes, when potential is measured between the middle two, and then from the bottom two electrodes, the admittances would be given by

$$
\begin{aligned}
& \Sigma Y_{2 j}=Y_{21}+Y_{22}+Y_{23} \\
& \Sigma Y_{3 j}=Y_{31}+Y_{32}+Y_{33}
\end{aligned} \quad \text { and }
$$

along the middle and bottom rows respectively and as shown in figure 2 .

Similarly for horizontal current drive and potential measurement between adjacent pairs of diagonal electrodes starting from the top are

$$
\begin{aligned}
& \Sigma Y_{\mathrm{i} 1}=\mathrm{Y}_{11}+\mathrm{Y}_{21}+\mathrm{Y}_{31}, \\
& \Sigma \mathrm{Y}_{\mathrm{i} 2}=\mathrm{Y}_{12}+\mathrm{Y}_{22}+\mathrm{Y}_{32} \\
& \Sigma \mathrm{Y}_{\mathrm{i} 3}=\mathrm{Y}_{13}+\mathrm{Y}_{23}+\mathrm{Y}_{33}
\end{aligned}
$$

and

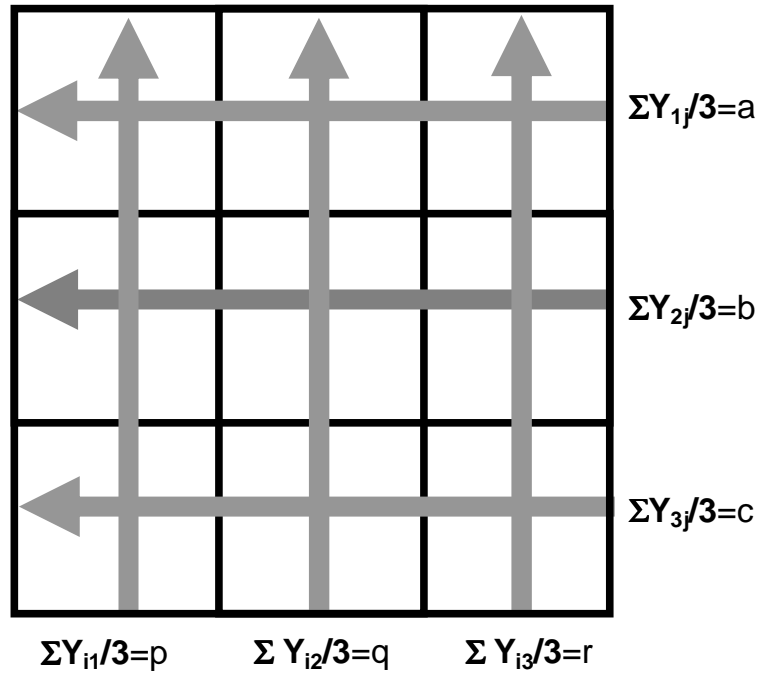

Fig. 3: Backprojecting $1 / 3^{\text {rd }}$ of the row and column admittance sums along respective rows and columns

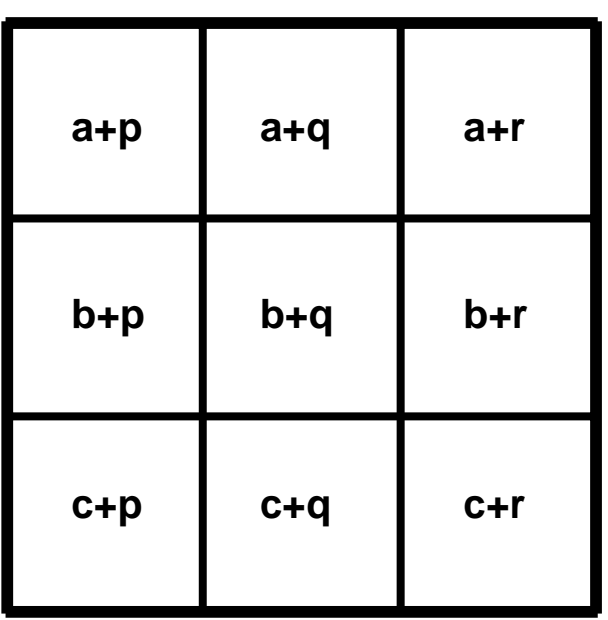

Fig. 4: Sum of the backprojected values in each pixel gives the image in PHI

along the three columns respectively as shown in figure 2 . The measured values would be the row and column sums as shown in the figure outside the matrix. 
Through backprojection of the measured sums along rows and columns (through a weighting factor of $\left.1 / 3^{\text {rd }}\right)$ it is possible to obtain the individual matrix values to some degree of approximation. For example, backprojection of $1 / 3^{\text {rd }}$ of $\Sigma Y_{1 j}$ and $\Sigma Y_{i 1}(a+p$ in figs 3 and 4) will have the value $\mathrm{Y}_{11}+\left(\mathrm{Y}_{12}+\mathrm{Y}_{13}+\mathrm{Y}_{21}+\mathrm{Y}_{31}\right) / 3$ where $\mathrm{Y}_{11}$ dominates compared to each of the other individual admittances. Therefore this backprojected value may be used as an estimate for $\mathrm{Y}_{11}$. Similarly other admittance values can be estimated through backprojection of all the six measured values as the sums $(a+p),(a+q), \ldots$ etc., as shown in figures 3 and 4. The image appears as square pigeon hole boxes, therefore the name Pigeon Hole Imaging (PHI) was chosen. Of course, the approximation for the values of each matrix position (or pixel value) is very crude, however, if the idea is to locate the position of a single object of a different conductivity in a uniform background, or of an object whose impedance changes between two sets of measurements while the background remains unchanged, then this method may provide an useful result.

\subsection{Phantom study}

Both 2D and 3D phantoms were employed for the purpose of measuring the sensitivity distribution of PHI. The phantoms basically used saline in appropriately configured containers. For 2D phantom, a container in the shape of a tray was made of plastic, as shown in figure 5. The electrodes, in the form of metallic pins, were driven vertically into the base so that their tops remain above the saline surface, from which connections were taken out. Saline was poured to a desired depth and a cylindrical object was placed at the desired positions to study the sensitivity at different pixel positions.

For 3D phantom a container was made out of clear acrylic plastic sheet, enclosed within a strong frame of mild steel angle-bars as shown in figure 6 . The internal base dimensions were $50 \mathrm{~cm}$ for each side and the height was $60 \mathrm{~cm}$. It was filled with saline to a depth of $50 \mathrm{~cm}$ making a phantom of dimensions $50 \mathrm{~cm} \times 50 \mathrm{~cm} \times 50 \mathrm{~cm}$. Seven potential measurement electrodes were fixed diagonally in the central region on one of the vertical walls of the tank. The vertical and horizontal separations between adjacent electrodes were $1.5 \mathrm{~cm}$ each. Any number of these electrodes, counting from the centre, could be used to produce $2 \times 2,3 \times 3$ up to $6 \times 6$ pixel images. Two sets of current drive electrodes were provided, an outer set at a separation of $24 \mathrm{~cm}$, and an inner set at a separation of $12 \mathrm{~cm}$. Insulating or conducting spheres were hung at desired positions inside the tank for PHI studies using this system. To facilitate such positioning, sliding frames with graduations for length were fixed on the top of the tank.

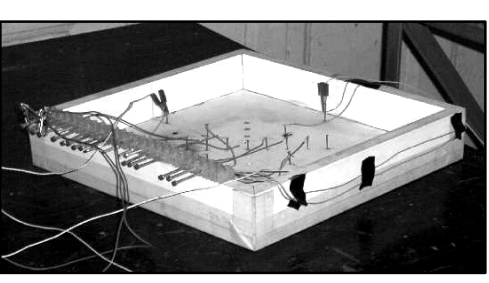

Fig. 5: 2D saline phantom

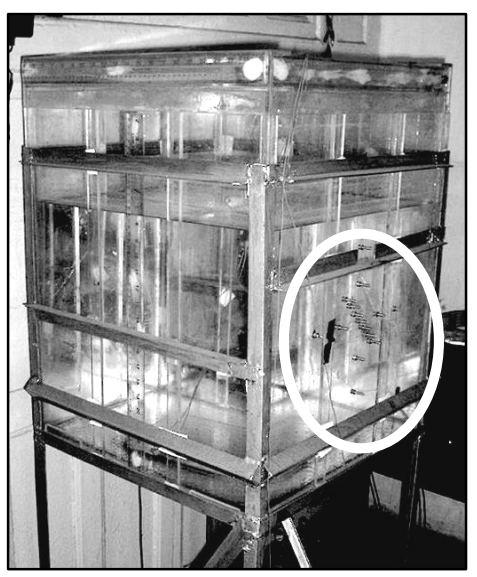

Fig. 6: 3D saline phantom. The electrodes are on the

\subsection{Measurement Methods}

The results obtained with the $2 \mathrm{D}$ and the $3 \mathrm{D}$ phantoms were found to be similar, so for brevity only a description of the measurements carried out using the 3D phantom at one depth from the electrode plane is given here. Instrumentation for a simple tetrapolar impedance measurement system, designed and developed by us, was used through manual switching of the appropriate electrodes, taking turns in acquiring data for different current and potential electrode combinations. The instrument provided a constant amplitude current drive at approximately $30 \mathrm{kHz}$. The potential developed between an adjacent pair of the three diagonal electrodes is amplified, filtered, rectified and smoothed to obtain a DC voltage, which is measured using a digital voltmeter. Since the current amplitude is constant only a voltage 
measurement was needed to get an impedance value, and its inverse provided the corresponding admittance.

Firstly for the $2 \times 2$ image an insulated sphere of $2.6 \mathrm{~cm}$ diameter was hung within the saline in the phantom so that its centre is located along the centre of the lower right matrix position formed by the 3 central potential electrodes. The centre of the sphere was at an inside distance of $1.3 \mathrm{~cm}$ from the electrode bearing wall so that the sphere just touched the wall with the electrodes. So this was in effect the depth of the centre of the object from the electrode plane. Current was driven through both the orthogonal outer pairs of electrodes and potential measurements were taken between each of the three adjacent potential electrodes for each current drive configuration. Analysis using simple backprojection as mentioned above gives the four pixel values of the image. Similar measurements were then taken without the sphere and analysis performed to obtain the background image. Then the pixel values of the background image were subtracted from the corresponding pixel values of the image with the object in the lower right pixel of the phantom as described above. This gave a difference image. The pixel values were then normalized to that having the highest value and these are shown in Fig. 7. A qualitative indication of the image is given by the gray shading. The white circle indicates the target position of the spherical object. It is clear that the highest pixel value corresponds to the position of the object, i.e., the image agrees very well with the target position of the object. The smearing of the image along the vertical and the horizontal directions are similar to star artifact, natural for this kind of image. Similar results were also obtained with the object positioned at all the other three pixels, and at greater depths from the electrode plane. However, the absolute pixel values were reduced for increasing depths which is natural to expect. Measurements were also carried out using a spherical conductor of the same size, and images with similar characteristics were obtained.

Measurements were then carried out using 7 electrodes along the diagonal making a 6x6 matrix. By hanging a spherical object to make its centre to correspond to the centre of a particular matrix position at a depth of $1.3 \mathrm{~cm}$ from the electrode plane a difference image was obtained in a similar way as described above for the 2x2 matrix. Similar difference images were then obtained with the object positioned at each of the 36 matrix positions, giving a total of 36 images.

\section{RESULTS AND OBSERVATIONS}

The $2 \times 2$ image obtained using a single object at the lower right matrix position was shown in figure 7.

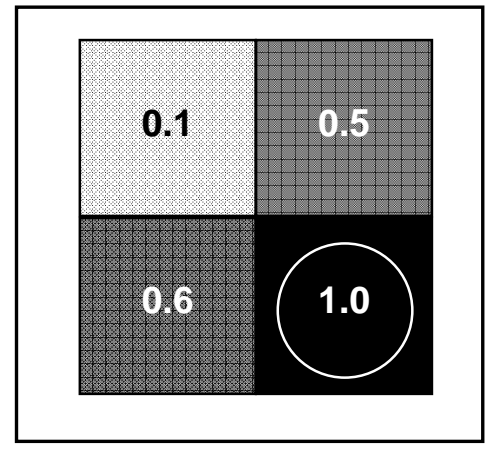

Fig. 7: Simple backprojected difference image (normalized) for an object at lower right pixel

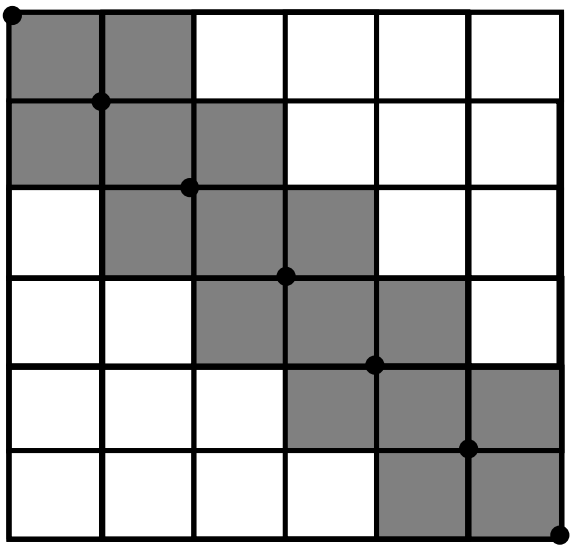

Fig. 8: Matrix positions where the image occurred at the correct position for a $6 \times 6 \mathrm{PHI}$ 
The target object position was indicated by a white circle and the pixel values have been normalized with respect to the highest value, as mentioned. It can be seen that the pixel with the highest value coincides with the target position. Pixel values of $60 \%$ and $50 \%$ were obtained in the adjacent horizontal and vertical positions which are natural to expect in any backprojection image. However, the position of the target object has been successfully identified by the highest value pixel. Similar results were obtained with the object placed in the other three matrix positions, and with both insulating and the conducting objects.

36 images were obtained for the 6x6 matrix PHI with a single object placed at each of the matrix locations in turn. The position of the highest pixel was indicated by a darkened square box. It was seen that for $16 \mathrm{PHI}$ images where the target objects were positioned adjacent to the electrode diagonal, the highest value pixel correctly represent the position of the target object. On the other hand for the rest 20 PHI images away from the electrode diagonal, the images are shifted slightly towards adjacent pixels. However, that at least for the 16 pixels the images are correct can be used to advantage in practical applications.

The 16 matrix positions for which the PHI images are correct are summarized in figure 8 through the dark shaded squares. The dark dots along the diagonal indicate the positions of the potential measuring electrodes.

\subsection{Image correction using a new smearing technique}

It was observed that where the images missed that actual position, there appeared a systematic shift or spreading of image (or smearing) almost enclosing the target position from both sides. Figure 10 shows the smearing of the images for all the 36 sets. Here only four levels of densities were used to shade the

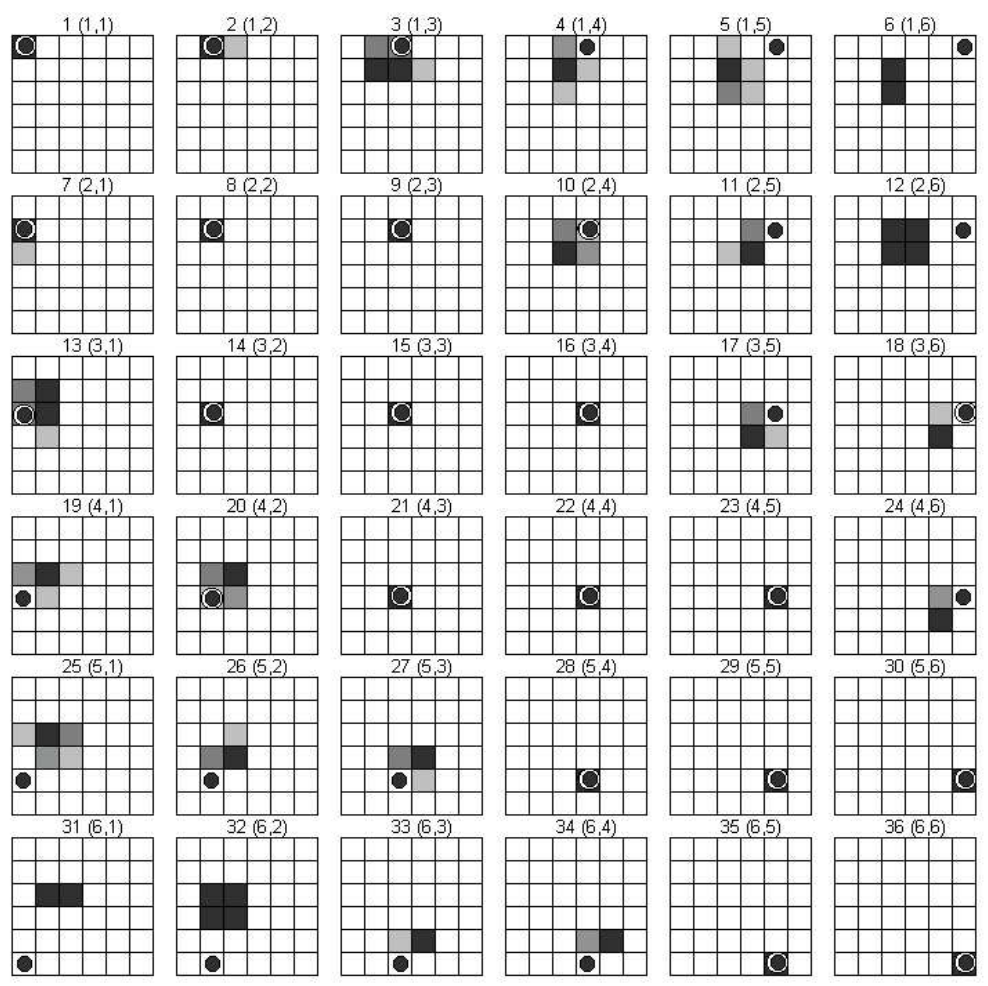

Fig. 9 Image smearing down to 50\% value of the maximum pixel value 
image pixels and only those pixels have been shaded which had pixel values greater than $50 \%$ of the maximum in each of the image. This led to an idea that the smearing pattern in the images may be used to correct the image positions. This can be justified, since the current lines and equipotential lines in the phantom will have definite patterns with the object in different pixel positions, therefore the smearing pattern may be unique for each pixel.

For convenience of identification, each image in Figure 10 has been given a reference number (1 to 36) together with the matrix element of the target position of the object. We can see that for images $1,8,15$, 22, 29 and 36 for which the target was placed along the diagonal of electrodes, there is no smearing of images. Similarly images 14, 21, 28 and 35 adjacent to the electrode-diagonal, there is no image smearing while for 7 along the same line parallel to the electrode-diagonal there is slight smearing in one direction only which can be neglected. In all the above image sets the image maximum correspond to the target object position well. Based on symmetry we can also say the same for the image 2, 9, 16, 23 and 30, which are on the other side of the electrode-diagonal.

Now, let us concentrate on Image 20 for target object at position $(4,2)$. The highest pixel value in the image occurs at $(3,3)$, with smearing going to adjacent $(3,2)$ and $(4,3)$ positions. Since we are not supposed to know the target object position beforehand we might be confused whether or not the densest image position at $(3,3)$ represents the target position. If we compare this image with Image 15 , which corresponds to the target position of $(3,3)$, we will see that there is no smearing in the latter image. Therefore just the presence of smearing, and that in two directions would give us a hint that in our system

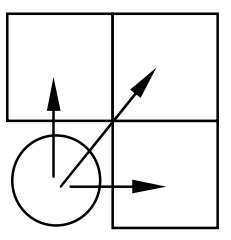

Fig. 10 Spreading or smearing of image from object at position $(4,2)$.

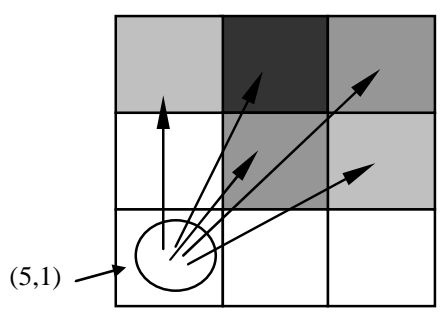

Fig. 12 Image smear pattern of object in position $(5,1)$

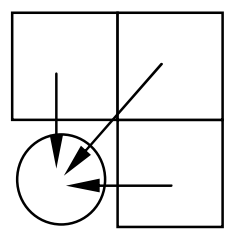

Fig. 11 Tracking back the object position from the smeared pattern.

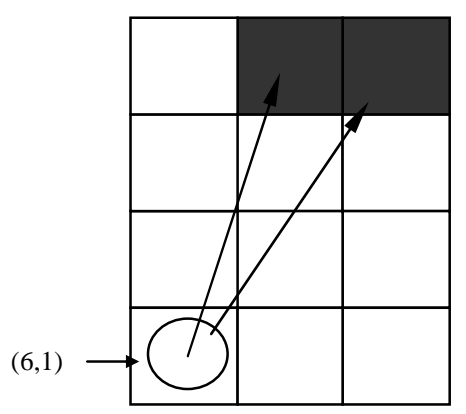

Fig. 13 Image smear pattern for object in position $(6,1)$

the maximum pixel value position is not the target object position. From our knowledge of the target position, $(4,2)$, it appears that the smearing pattern has enclosed its position from both sides as shown in Fig.10. It appears in this figure that the object has spread out its image northwards, northeastward and 
eastward. Therefore, such a smear pattern in the image would tell us that the object position is different from the image maximum, and that the target position can be deciphered by back-tracking the expected image shift as shown if Fig 11.

Images 13, 27 and 34 of Fig 9, which aligns with image 20 along a line parallel to the electrode-diagonal observe a similar pattern of image smearing from which the target position can be obtained, using the same technique as described above. Image 34 has smearing only westwards. Besides image 29, of Fig. 9 , with image maximum at the same position $(5,5)$ there is no smearing at all and therefore is different from Image 34 .

Thus we can correct the target object positions for images 13, 20, 27 and 34 using this 'image smear pattern' technique. Similar patterns of image smear can be observed for images 19, 26 and 33. Of course it can be seen that the pixel values for these images are very small since the target object is far away from the diagonal. Therefore errors in measurements are increased. For images 25, 32 and 31, of Fig.5, the pixel values are very small and it is difficult to make any comments on the image smear pattern. However, some pattern may be identified in images 25 and 31 as shown in Fig. 12 and 13.

Since these pixel values are too small we are not claiming these patterns to give useful results, but are presented to give some indications only. For the target positions on the other side of the electrodediagonal we get symmetrical results.

The images in Figure 9 can therefore be classified by the following Image Rules:

Summarizing the above results we can state the following rules for imaging of a single object using PHI, where images are drawn down to $50 \%$ of the maximum pixel value in each image. The rules are stated referred to group identifications of target positions given in the previous section.

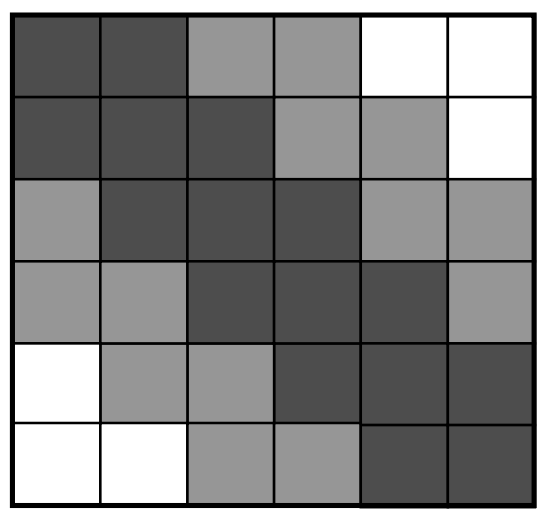

Fig. 14. The total matrix positions for which PHI image localizes a single object

1. 16 target positions whose centers are within 0.5 pixel-diagonal of the electrode-diagonal, the image does not smear out and the image maximum corresponds to the target position.

2. 14 target positions whose centers are between 0.5 and 1.5 pixel-diagonal of the electrodes-diagonal, the image smears out asymmetrically in two perpendicular directions from the image maximum. In this case the image maximum does not correspond to the target position, which can be identified by the matrix position that is enclosed or bracketed by the smeared image pattern, whose center is 0.5 pixel-diagonal away from the center of the image maximum.

3. 6 target positions whose centers cannot be ascertained by the present work as it does not show any symmetric pattern, nor was the instrumental sensitivity adequate. The patterns indicated in this work for these two groups will need further work for confirmation. 
Thus using the above image rules we can determine the positions of a single object in 30 out of the 36 matrix positions in a PHI system with 7 potential electrodes as shown by the shaded positions in Fig.14, the dark ones indicating where the targets are correctly imaged and the gray ones indicating where the target can be determined using the correction rules presented here based on image smearing.

\section{DISCUSSION}

The present idea of PHI grew out of the diagonal placement of several potential measuring electrodes in a 6-electrode FIM. Having more numbers of electrodes along this diagonal allows more small adjacent zones to be focused.

In the present work a detailed experimental verification of the concept of PHI was attempted, starting with a simple $2 \times 2$ matrix, and then, with a higher $6 \times 6$ matrix, employing three and seven diagonal electrodes respectively. The concept may be extended further to higher order matrices by increasing the number of diagonal electrodes, however, the errors away from the electrode diagonal will increase. For the 6x6 matrix, it has been shown that correct target localization is possible within most of the imaged area. In many physiological and diagnostic applications an approximate location of a target object is known. Its exact location can then be ascertained using PHI with suitable placement of electrodes, and a $6 x 6$ matrix should be reasonable maximum size from a practical point of view.

In a tetrapolar impedance measurement system, the current density due to the current drive electrodes, and perturbation of equipotentials at the potential measuring electrodes are the two things that determine the sensitivity of the measurement at a particular point. This agrees with the experimental results obtained in this work, and therefore the pixels adjacent to the electrode diagonals have produced correct images. It should also be noted that if two objects to be imaged are in close proximity, their combined contribution in bending the equipotentials will give rise to a smeared out image and the positions of the objects may not be resolved. However, if the objects are widely apart and if they can be located approximately around a diagonal of the PHI electrode configuration, then resolving the images may be possible. One advantage of the PHI technique is that by increasing or decreasing the spacing of potential measuring electrodes it is possible to choose a desired spatial resolution around a desired region of interest. Therefore, if there are multiple objects at shallow depths, appropriate choice of electrode placement and spacing may be utilized to image the objects separately through a single procedure.

It can be visualized from the results of the present study that by having additional potential measuring electrodes along the other diagonal, images in at least 12 further pixels may be obtained correctly. It needs to be seen whether the remaining 8 pixels at the edges of the sides are correctly imaged in this procedure. However, this will obviously need more electrodes than the single diagonal electrode configuration. Again, looking at the images in figure 8, a systematic pattern in the shift of the images can be observed for the positions not giving the correct image. It may be possible to devise ways to correct these images through analytical or other techniques.

Though the procedure for PHI is ideally meant for a 2D study, the technique may well be applied to study objects down to certain depths from a surface in a volume conductor since currents flow in 3D as well. As mentioned in the methods section, measurements with the target object at a few shallow depth values were carried out and these gave similar images, although with reduced values of pixels.

The advantages of PHI system are that it can be designed at relatively low cost and has no known significant hazard. Moreover, the system can easily be adapted for dynamic functional studies. Being simpler than EIT, it can be applied to cases where a crude image is all what is needed, particularly in cases where the admittance (or impedance) changes at one point, or at a few widely spaced regions so that 
the images do not interfere with one another. Even with potential electrodes along both diagonals, this system will be much simpler compared to EIT, both in hardware and software.

An advantage of PHI is that the resolution of a zone of interest can be increased by decreasing the spacing of the potential measuring electrodes while keeping the current electrodes unchanged. Again the depth sensitivity can be changed by changing the separation of the current drive electrodes.

In conclusion, it may be said that PHI has added another new imaging modality to the existing ones that may be used to advantage in certain applications.

\section{REFERENCES}

1. Barber D.C., Brown B.H. and Freeston I.L. Imaging spatial distributions of resistivity using applied potential tomography. Electron. Lett. 19:933-5, 1983.

2. Barber D.C. and Brown B.H. Applied potential tomography. J. Phys. E: Sci. Instrum. 17:723-733, 1984.

3. Rabbani K.S. and Kabir H. Studies on the effect of the third dimension on a two dimensional Electrical Impedance Tomography system. Clin. Phys. \& Physiol. Meas. 12:393-402, 1991.

4. Rabbani K.S., Hassan M. and Kiber A. 3D object localisation using EIT measurement at two levels. Physiol. Meas.17:189-199, 1996

5. Rabbani K.S., Sarker M., Akond M.H.R. and Akter T. Focused Impedance measurement (FIM) - a new technique with zone localization. Proceedings, X International Conference on Electrical Bioimpedance, Spain. 31-34, 1998.

6. Rabbani K.S., Sarker M., Akond M.H.R. and Akter T. Focused Impedance Measurement (FIM) - a new technique with improved zone localization. Ann. N.Y. Acad. Sc. 873: 408-420, 1999.

7. Kadir M.A., Baig T.N. and Rabbani K.S. Application of 6-electrode Focused Impedance Method (FIM) to study lungs ventilation. Proceedings (on-line), 10th International Conference on Biomedical Applications of Electrical Impedance Tomography (EIT2009), UK, 2009.

8. Afroj K, Alam N, Rahman M and Rabbani K S, Pigeon hole imaging (PHI) - an electrical admittance backprojection technique, Bangladesh J. of Medical Physics, 3: 7-13, 2004 